\title{
ANDROID BASED FUSED LOCATION PROVIDER API FOR LOCALIZATION ACCURACY
}

\author{
Ashpaqahmad Nadaf ${ }^{1}$ and Prof. B K Srinivas ${ }^{2}$
}

\begin{abstract}
In fast moving world, Location accuracy become an important issue. Google provides API as Fused Location Provider which manages the accuracy, battery performance between GPS and Network provider. This paper details the mechanism which can be used to get best device's location with high accuracy using fused location API provider.

Keywords - Android, Fused Location API, Real tracking, Location, latitude and longitude
\end{abstract}

\section{INTRODUCTION}

The rapid development and enhancement in the smart phone devices becomes the important role in the moving world especially in the location accuracy for navigation based applications. This paper illustrate in improve location accuracy using Fused Location Provider API. There are 3 ways in which we can get real time locations in smart phones.

1. GPS based location provider in android using LocationManager.GPS_PROVIDER that makes use of satellite. It gives high accuracy of current location but need continuous power supply.

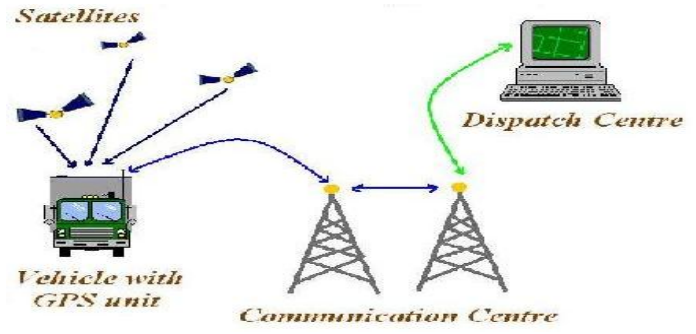

Figure1. GPS based location provider

2. Network based location provider using LocationManager.NETWORK_PROVIDER that makes use of Cellular Network points but the accuracy of using this is lesser than GPS. One advantage is the battery consumption is low.

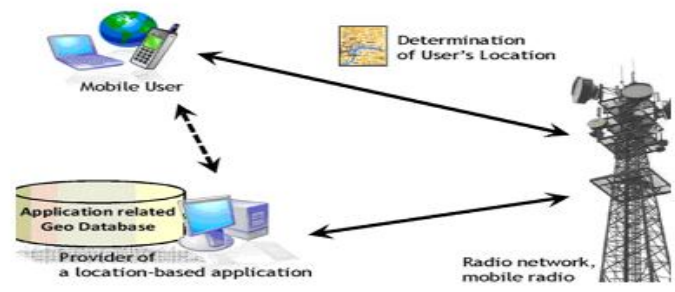

Figure2. Network based location provider

${ }^{1}$ Department of Information Science \& Engg., RVCE, VTU Bengaluru, Karnataka, India

${ }^{2}$ Department of Information Science \& Engg., RVCE, VTU Bengaluru, Karnataka, India 
3. Passive Location Provider determines the location without need the application to trigger the providers using LocationManager.PASSIVE_PROVIDER. It work by some other services or application, passively give updating of the location to the apps which uses it but the accuracy is not so go'od compare to the above two providers.

\section{LITERATURE SURVEY REVIEW}

In the starting versions of the android have Location APIs. Location tracking is a simple but getting accuracy is difficult. Android APIs starts from Location manager and then APIs using Google Play Services.

Location Manager Provider API uses above mentioned three types for finding the location. But the performance, accuracy is not so good[3][4]. Google developed API called Google location service API that uses Fused location provider. Here we can get accuracy because getting location service from system wide which keeps updating. Google Play Service Client is used to connect clients or your fragment (Activity). Immediately trying to get location because the Fused location provider maintains one process in background when at least one client connects, which calls get Last Location method in onConnected method. Here the result may be null. So to solve this we need to implement Google provided interface com. google. gms. location. LocationListener by using this we can get periodic updates and it will switch off upon getting first update using onLocation Changed()callback[6].

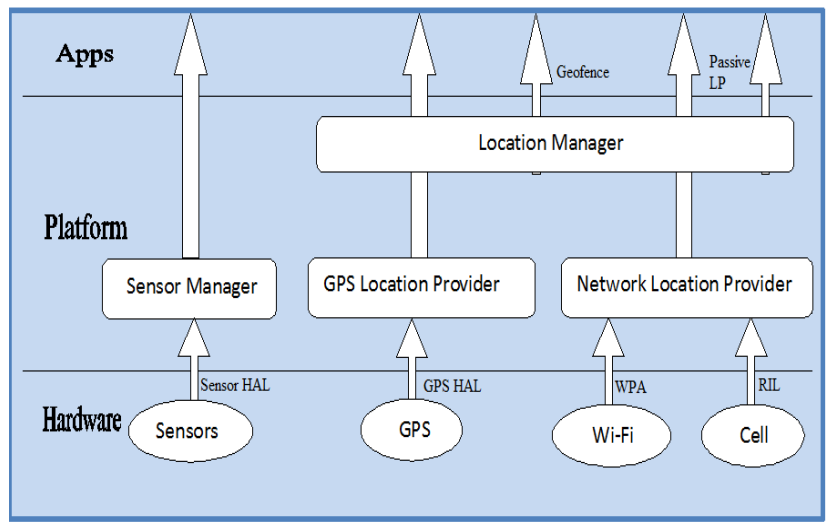

Figure3. Location Manager Provider API

In Figure 3 the problem in the API called Location Manager provider was

1. developer to use the network location provider where GPS is not able to work in indoors

2. Battery decreases when to switch for exact location provider[7]

\section{Proposed Location Provider}

\section{Fused Location Provider}

Fused location provider is help to get the location updates periodically. When user is leaving or enetering into some are it will notify (this type of feature is called geofencing). Using the geofencing an apps can track up to hundread targets. To avoid distant tracking targets geofencing uses cellular location data [1][2]. 


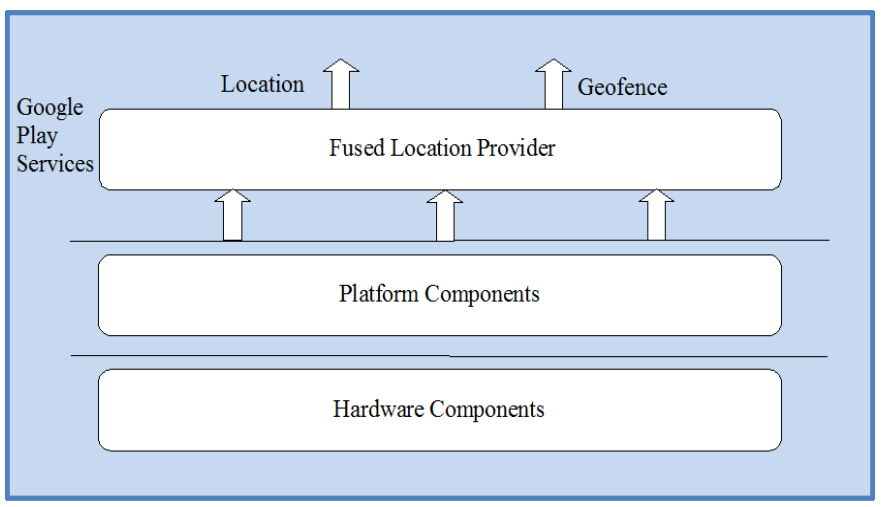

Figure4. Fused Location Provider API

To adjust the required location upadates frequency, there are the different sensors that are used by Fused Location The five User states or activities are

1. in_vehicle

2. on__bicycle

3. on_foot

4. _still

5. _tilting

In some times actions of the user cannot be determined thus the state is UNKNOWN.

Applications which uses the location services must request for permissions in terms of location. Usally Android offers 2 permissions: ACCES_FINE_LOCATIONS, ACCES_CCOARSE_LOCATIONS. Choice of permissions determines the location accuracy returned by application programming interface[6].

Add uses-permission element in manifest file depending on your choice of location permission shown in Figure 5. To connect to Google Location service API, first it is required to create an instance of the Google Play services API client i.e to add Location service API GoogleApiClient.Builder class is used which is shown in the Figure 6 code snippet[8].

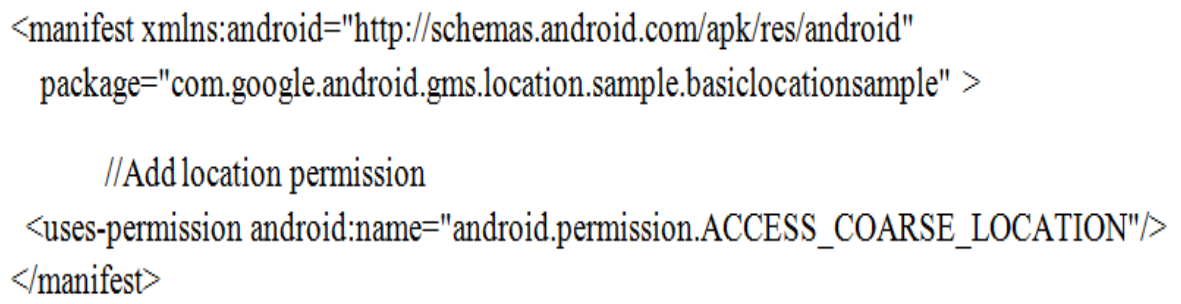

Figure 5: Android manifest file to add location permission

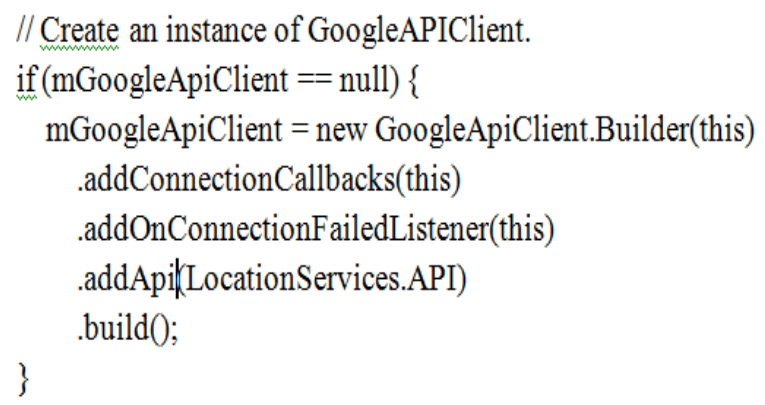

Figure 6: Android manifest file to add location permission 
To connect, Use a method onStart() to call connect() and to disconnect, onStop() method to call disconnect(). The following Figure 7 code snippet shows an example of how to use both of these methods[9].

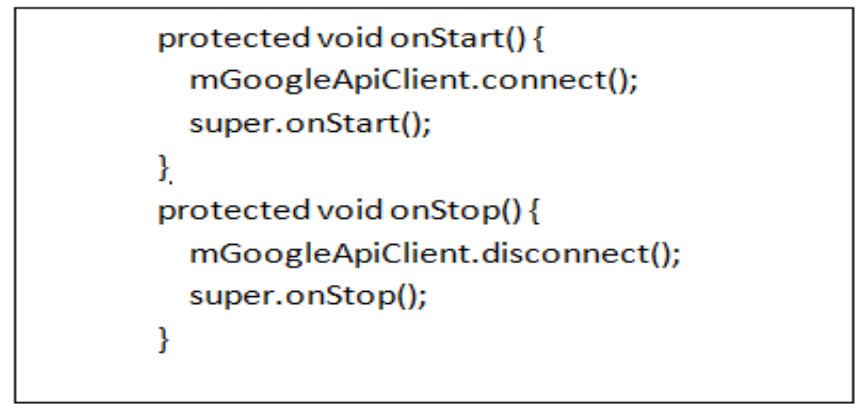

Figure 7: Android manifest file to add location permission

\section{RESULTS}

Google Fused Location provider Can be used in the following 3 steps

1. Connecting Location Client to API(Google). Get location After connecting locationClient.

2. getting current location of the user.

3. Continous updation of the location at particulars time of interval or the particular distances or may be both.

The following Table 1 shows the Google tested the location accuracy using Fused location provider API vs Location manager in the google nexus.

Table 1. Location problem solved by Google with user priorities

\begin{tabular}{|c|c|c|c|}
\hline Priority & $\begin{array}{c}\text { Typical } \\
\text { location } \\
\text { update } \\
\text { interval }\end{array}$ & $\begin{array}{c}\text { Battery } \\
\text { drain per } \\
\text { hour (\%) }\end{array}$ & Accuracy \\
\hline HIGH_ACCURACY & 5 seconds & $7.25 \%$ & $\sim 10$ meters \\
\hline BALANCED_POWER & 20 seconds & $0.6 \%$ & $\sim 40$ meters \\
\hline NO_POWER & N/A & Small & $\sim 1$ mile \\
\hline
\end{tabular}

By looking to Table 1 we can list some Advantages of Using Fused API is

1. Simple APIs: Provides location with "low power" and "high accuracy rather than worrying about area suppliers.

2. Immediately Available: Gives our applications quick access to the best, latest area.

3. Power-Efficiency: Meets an extensive variety of necessities, from frontal area utilizes that need profoundly precise area to foundation utilizes that need intermittent area refreshes with insignificant power affect.

4. Versatility: Meets an extensive variety of necessities, from frontal area utilizes that need profoundly precise area to foundation utilizes that need intermittent area refreshes with insignificant power affect.

\section{IV.CONCLUSION}

Now getting accuracy of the location is more important due to increasing the number of user and problem. So tracking is main theme. This paper reviews, Fused location provider API developed by Google provides more accuracy by switching to the available location provider than the previous Location manager provider APIs. Hence developing the applications using this Fused location API provides high accuracy. 


\section{REFERENCES}

[1] Google, "Fused Location Provider API", https://developer.android.com/training/location/retrievecurrent.html\#play-services.

[2] Google,"Fused_Location_API",https://developers.google.com/android/reference/com/google/android/gms/location/FusedLocationProvider Api

[3] Google, "Location manager provider API", https://developer.android.com/reference/android/location/LocationManager.html

[4] Google, "Location manager provider API", http://javapapers.com /android/get-current-location-in-android/

[5] Google, "Fused Location Provider API", http://blog.lemberg.co.uk /fused-location-provider

[6] Stack Overflow "Fused location and location manager", http://stackoverflow.com/questions/33022662/androidlocationmanager-vs-googleplay-services.

[7] Google, "Location manager provider API", https://developers_android_com/training/locations/change-locations-settings.html.\#connect

[8] Google, "Google's Fused Location API for Androids",http://www.tothenew.com/blog/googles-fused-location-api-for-android/

[9] Bhaskar, Nupur, "localization accuracy improvement in android by using Fused Location API with help of Kalman Filter", 2016 International conf. Computer Communication and Informatics (ICCI),2016,pp 1 - 4 